\title{
Can Event-Related Potentials Evoked by Heel Lance Assess Pain Processing in Neonates? A Systematic Review
}

\author{
Yui Shiroshita ${ }^{1, *}$, Hikari Kirimoto ${ }^{2} \oplus$, Mio Ozawa ${ }^{1} \oplus$, Tatsunori Watanabe ${ }^{2}$, Hiroko Uematsu $^{3}$, Keisuke Yunoki $^{2}$ \\ and Ikuko Sobue ${ }^{1}$ \\ 1 Division of Nursing Sciences, Graduate School of Biomedical and Health Sciences, Hiroshima University, \\ Hiroshima 734-8553, Japan; ozawamio@hiroshima-u.ac.jp (M.O.); sobue@hiroshima-u.ac.jp (I.S.) \\ 2 Department of Sensorimotor Neuroscience, Graduate School of Biomedical and Health Sciences, \\ Hiroshima University, Hiroshima 734-8553, Japan; hkirimoto@hiroshima-u.ac.jp (H.K.); \\ twatan@hiroshima-u.ac.jp (T.W.); d205546@hiroshima-u.ac.jp (K.Y.) \\ 3 School of Nursing, University of Human Environments, Aichi 474-0035, Japan; hirokouematsu0@gmail.com \\ * Correspondence: shiroshita@hiroshima-u.ac.jp; Tel.: +81-82-257-1761
}

Citation: Shiroshita, Y.; Kirimoto, H.; Ozawa, M.; Watanabe, T.; Uematsu, H.; Yunoki, K.; Sobue, I. Can Event-Related Potentials Evoked by Heel Lance Assess Pain Processing in Neonates? A Systematic Review. Children 2021, 8, 58. https://doi.org/ $10.3390 /$ children 8020058

Academic Editor: Sari Acra Received: 15 November 2020 Accepted: 16 January 2021 Published: 20 January 2021

Publisher's Note: MDPI stays neutral with regard to jurisdictional claims in published maps and institutional affiliations.

Copyright: (C) 2021 by the authors. Licensee MDPI, Basel, Switzerland. This article is an open access article distributed under the terms and conditions of the Creative Commons Attribution (CC BY) license (https:// creativecommons.org/licenses/by/ $4.0 /)$.

\begin{abstract}
To clarify the possibility of event-related potential (ERP) evoked by heel lance in neonates as an index of pain assessment, knowledge acquired by and problems of the methods used in studies on ERP evoked by heel lance in neonates were systematically reviewed, including knowledge about $A \delta$ and $C$ fibers responding to noxious stimuli and $A \beta$ fibers responding to non-noxious stimuli. Of the 863 reports searched, 19 were selected for the final analysis. The following points were identified as problems for ERP evoked by heel lance in neonates to serve as a pain assessment index: (1) It is possible that the ERP evoked by heel lance reflected the activation of $A \beta$ fibers responding to non-noxious stimuli and not the activation of $\mathrm{A} \delta$ or $\mathrm{C}$ fibers responding to noxious stimulation; (2) Sample size calculation was presented in few studies, and the number of stimulation trials to obtain an averaged ERP was small. Accordingly, to establish ERP evoked by heel lance as a pain assessment in neonates, it is necessary to perform a study to clarify ERP evoked by A $\delta$ - and C-fiber stimulations accompanied by heel lance in neonates.
\end{abstract}

Keywords: heel lance; infant; event-related potential; pain assessment tool

\section{Introduction}

Neonates in neonatal intensive care unit (NICU) are exposed to repetitive painful procedures in the period of complex and rapid brain development [1]. Neonates are particularly sensitive to unanticipated external stimuli, and repetitive exposure to painful medical procedures can have an effect on the development of the central nervous system [2]. Cohort studies demonstrated that exposure to painful procedures can reduce the volume of white and gray matters in preterm infants [3] and that this adverse effect lasts until adolescence [4-6]. Repetitive exposure to painful procedures in neonates can also have an impact on behavioral abnormalities [7,8], as indicated by reduced cognitive score $[7,8]$, as well as internalizing behaviors [9], and these adverse effects last until young adulthood [10-14]. Collectively, these studies suggest that neonates are put at significant risk by being subjected to repetitive painful procedures. Although the mechanism of how pain causes damage to the central nervous system in neonates remains unknown, a study in 1-week-old rats suggested that exposure to repetitive pain can alter the production of proteins involved in the processes of development by inducing apoptosis of crucial neurons [15].

Heel lance is one of the most commonly performed procedures associated with pain [16,17]. Multiple interventions such as pacifiers, facilitated tucking, holding, and music are used alone or in combination to reduce the impact of the pain associated with heel lance in neonates [18-25]. 
Most studies that examined pain relief strategies for heel lance used either the premature infant pain profile (PIPP) or premature infant pain profile-revised (PIPP-R) as a measure of pain. PIPP consists of two physiological measures (heart rate and oxygen saturation) and three behavioral measures (brow bulge, eye squeeze, and nasolabial furrow), and the score is corrected for gestational age (GA) and behavioral state [26,27]. PIPP-R further revised the weights to adjust for GA and behavioral state [28,29]. Both PIPP and PIPP-R were shown to be reliable and valid [26,28-30]. However, a study demonstrated that PIPP has poor sensitivity in detecting responses to low-intensity noxious stimuli [31]. Thus, an objective and quantitative measure of pain is needed to properly assess the pain associated with heel lance in neonates to examine the effectiveness of various pain relief strategies.

Event-related potential (ERP) evoked by nociceptive stimulation has recently been attracting attention as an index of pain assessment in neonates [32-36]. Noxious stimuli activate peripheral nociceptors and pain-sensing neurons in the skin, and myelinated A $\delta$ and unmyelinated $C$ fibers are the main peripheral nociceptors [37-39]. A $\delta$ fibers respond to rapid, pricking, and localized pains, while $\mathrm{C}$ fibers respond to diffuse burning or aching sensations [37,40-43]. Noxious stimuli detected by peripheral nociceptors are transmitted to the cerebral cortex via the spinal cord, brainstem, and thalamus [44,45]. ERP detects changes in electrical activity generated by neurons in the brain.

In adults, it has become possible to selectively stimulate $A \delta$ and $C$ fibers with intraepidermal electrical stimulation (IES) and laser stimulation and to record evoked potentials [46]. Furthermore, it has been reported that the amplitude of ERP evoked by IES and laser stimulation decreased after analgesic administration in adults $[47,48]$. Thus, ERP evoked by nociceptive stimuli can be used as a tool for assessing the efficacy of pain management. In regard to neonates, ERP evoked by heel lance was initially reported in 2010 [49], and it was considered to be an objective measure of pain in neonates being unable to express pain in language. However, to our knowledge, there has been no systematic review of the results of ERP evoked by heel lance in neonates. Only a scoping review [50] and unsystematic review articles [32-34,36] have been reported previously. In the scoping review, the absence of sample size calculation and problems with statistical analysis have been pointed out [50]. A systematic review in this area will help to evaluate the existing evidence and provide a platform to identify future research needed to extend our knowledge.

In this study, to clarify the possibility of ERP evoked by heel lance in neonates as an index of pain assessment, knowledge acquired by and problems of the methods used in studies on ERP evoked by heel lance in neonates were systematically reviewed, including knowledge about $A \delta$ and $C$ fibers responding to noxious stimuli and $A \beta$ fibers responding to non-noxious stimuli. A better understanding of the mechanisms underlying pain responses in the central nervous system of neonates will lead to the development of an appropriate measure of pain in neonates.

\section{Materials and Methods}

\subsection{Statement on Review}

The present review was performed in accordance with the Preferred Reporting Items for Systematic Reviews and Meta-Analyses (PRISMA) guidelines [51,52].

\subsection{Search Strategy}

PubMed, CINAHL, PsycINFO, Scopus, and CENTRAL were searched until January 2021 with no start date. Keywords for the search were ("heel lance" OR "heel stick" OR "heel prick" OR "heel puncture" OR "pain") AND ("EEG" OR "ERP" OR "electroencephalogram" OR "event related potential") AND ("neonate" OR "infant" OR "newborn"). Studies that met the following criteria were included: (1) studies that evaluated whether ERP was appropriate as an objective measure of nociception induced by heel lance in neonates, or (2) randomized controlled trials (RCTs) or non-RCTs that used ERP to determine the efficacy of pain relief strategies to reduce pain associated with heel lance in neonates. Studies were 
also included if they evaluated ERP itself or in comparison to other measures. Both preterm and full-term infants were included in our definition of neonates.

\subsection{Selection Criteria}

The following inclusion criteria were used based on the PICOS model. (P) population: preterm and full-term infants admitted to NICU; (I) intervention: whether ERP is appropriate for pain evaluation; (C) comparison: with another pain assessment tool (PIPP, behavioral state, near-infrared spectroscopy (NIRS), and/or electromyography (EMG)); (O) outcomes: ERP responses; (S) study design: RCT and non-RCT. Studies were excluded from the review if they (1) assessed pain that is not associated with heel lance, (2) used pain assessment tools other than ERP, or (3) were written in a language other than English.

\subsection{Study Selection Process}

\subsubsection{Primary Screening}

Two independent investigators (Y.S. and I.S.) screened the literature based on the title and abstract, and duplicates were removed from a list of studies. When the two investigators disagreed on the inclusion of a study, they discussed it. When it could not be resolved by discussion, a third investigator (H.U.) made the decision on whether to include or exclude the study.

\subsubsection{Secondary Screening}

Based on the primary screening, two investigators (Y.S. and H.U.) independently identified studies that met the inclusion criteria. When the two investigators disagreed on the inclusion of a study, they discussed it. When it could not be resolved by discussion, a third investigator (I.S.) made the decision to include or exclude the study.

\subsection{Assessment of the Methodological Quality of the Studies}

The quality of the studies was assessed independently by two investigators (Y.S. and H.U.). Some of the studies were discussed between the two investigators, and a third investigator (M.O.) made the decision to include or exclude the study when needed. In four studies investigating whether ERP can be used for pain assessment in neonates by recording brain activities in response to heel lance required for particular treatments and to harmless tactile stimuli, the study protocol was regarded as observational by the authors [53-56]. For the purpose of our review, these pain-inducing procedures were considered as interventions, and we categorized these four studies, in addition to 10 other studies with no study design information, as non-RCTs (comparative and non-comparative study design).

The quality of non-RCTs was evaluated using the methodological index for nonrandomized studies (MINORS) [57]. MINORS assesses the methodological quality of non-RCTs and is applicable to both comparative and non-comparative studies. It consists of the following 12 items, of which the first eight are applicable to non-comparative studies while all are applicable to comparative studies: (1) a clearly stated aim; (2) inclusion of consecutive patients; (3) prospective collection of data; (4) endpoints appropriate to the aim of the study; (5) unbiased assessment of the study endpoint; (6) follow-up period appropriate to the aim of the study; (7) loss to follow up less than $5 \%$; (8) prospective calculation of the study size; (9) an adequate control group; (10) contemporary groups; (11) baseline equivalence of groups; and (12) adequate statistical analyses. Each item is scored between 0 and 2 (0: not reported; 1: reported but inadequate; or 2: reported and adequate), and the global ideal scores are 24 and 16 for comparative and non-comparative studies, respectively. It is a reliable and validated system [57] and has been used in various reviews [58].

The methodological quality of RCTs was evaluated using the modified Jadad quality scale [59-61]. Its evaluation is based on whether a study includes the description of the following six items: (1) randomization; (2) double-blinding; (3) withdrawals and dropouts; 
(4) inclusion and exclusion criteria; (5) method used to assess adverse effects; and (6) statistical analyses used. Each item is scored either 0 (no) or 1 (yes), with a positive answer getting a point. Furthermore, an additional point is given for items 1 and 2 if the methods of randomization and blinding are appropriate, and a point is deducted if the methods are inappropriate. Studies with overall scores of $0-3$ and 4-8 are considered to be of low and high quality, respectively $[60,62]$. The modified Jadad quality scale has been used in a number of systematic reviews [60], including a systematic review on the topic of pain assessment scale in children [63].

\subsection{Data Extraction}

Data were extracted from studies independently by two investigators (Y.S. and H.U.). The two investigators discussed differences in data analysis when needed, and a third investigator (K.Y.) was involved in the decision-making process in some cases. The following information was extracted from non-RCTs: age at birth of participants; age at time of the study of participants; number of participants; stimulation; outcomes, and results. Similarly, the following information was extracted from RCTs: age at birth of participants; age at time of the study of participants; number of participants; stimulation; intervention; outcomes, and results.

\section{Results}

\subsection{Study Selection}

A total of 863 studies were extracted from the databases, and 19 were included in the review (Figure 1). Five of those studies were RCTs [64-68] and the remaining 14 were non-RCTs. Among the non-RCTs, five were comparative studies $[53,55,69-71]$ and the remaining nine were non-comparative studies $[31,49,54,56,72-76]$.

\subsection{Study Characteristics}

Table 1 lists the MINORS scores for 14 non-RCTs and Table 2 lists the modified Jadad scale score for 5 RCTs. Three RCTs were considered to have high methodological quality. Tables 3 and 4 summarize the studies included in the review. The GA of the study subjects ranged from 23 to 42 weeks at birth and 28 to 47 weeks at the time of the study. A total of 854 neonates admitted to an NICU were included in the analysis. One of the studies included only preterm infants [53], 11 included both preterm and full-term infants $[49,55,56,64,68-70,72,74-76]$, and 6 included only full-term infants [31,54,65-67,71]. One study did not indicate the GA of the study subjects [73].

Four studies examined the response to heel lance alone $[53,64,70,75]$ and nine examined the response to heel lance and tactile stimulus (contact of a heel lance device against the skin [49,66,71,74,76], rubber bung [69], tendon hammer [72-74], air puff [54], or cold puff [54]). Other studies examined the response to the following stimuli in addition to heel lance: tactile stimulation (contact of a heel lance device against the skin) and auditory stimulation [56], experimental noxious stimulation (pinprick) [31,65], experimental noxious stimulation (pinprick) and blood sampling [67], experimental noxious stimulation (pinprick), tactile stimulation (tendon hammer), visual stimulation, and auditory stimulation [55], and tactile stimulation (contact of a heel lance device against the skin) and retinopathy of prematurity screening examination [68].

The outcome measures included in the studies were as follows: ERP alone [49,64,69-72]; ERP and either PIPP or PIPP-R [67,68,76]; ERP, PIPP, and EMG [31,66]; ERP, PIPP, and heart rate [55]; ERP, PIPP, facial expression, heart rate, and oxygenation [56]; ERP and EMG [65]; ERP and NIRS [74]; ERP, EMG, NIRS, ECG, behavioral response, and autonomic responses (heart rate, oxygen saturation, respiratory rate, and cardiovascular activity) [73]; ERP, facial response, and heart rate [64]; ERP and crying [54]; ERP, PIPP, salivary cortisol, and heart rate variability [75], and ERP and behavioral indicators of infant pain (BIIP) [53]. 

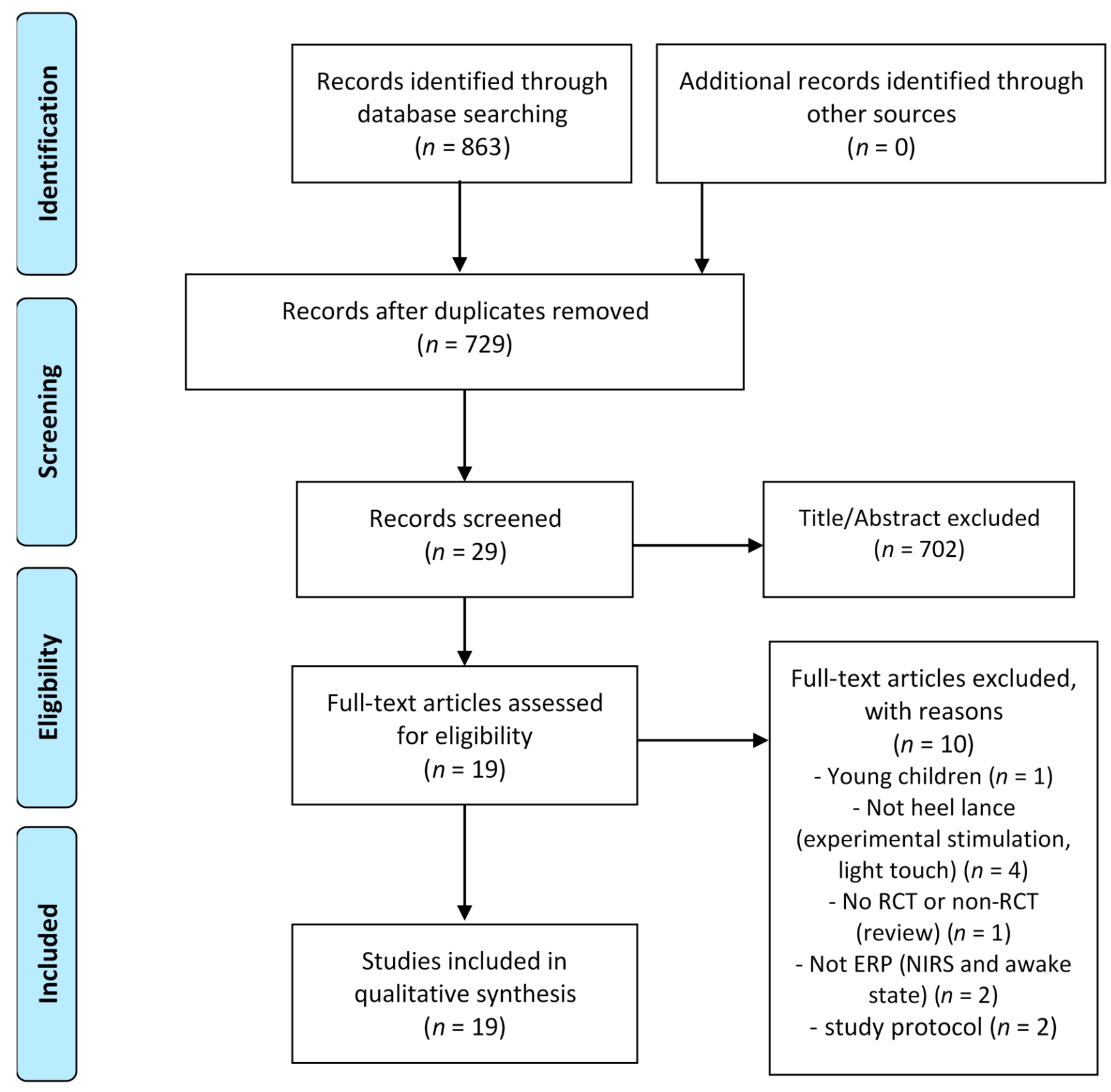

Figure 1. Flow chart of included article. 
Table 1. Assessment of the methodological quality of the non-randomized controlled trial (non-RCT) (methodological index for non-randomized studies (MINORS) score).

\begin{tabular}{|c|c|c|c|c|c|c|c|c|c|c|c|c|c|c|}
\hline Author, Year & $\begin{array}{c}\text { Study } \\
\text { Design }\end{array}$ & $\begin{array}{l}\text { A Clearly } \\
\text { Stated Aim }\end{array}$ & $\begin{array}{l}\text { Inclusion of } \\
\text { Consecutive } \\
\text { Patients }\end{array}$ & $\begin{array}{c}\text { Prospective } \\
\text { Collection } \\
\text { of Data }\end{array}$ & $\begin{array}{l}\text { Endpoints } \\
\text { Appropriate } \\
\text { to the Aim } \\
\text { of the Study }\end{array}$ & $\begin{array}{l}\text { Unbiased } \\
\text { Assessment } \\
\text { of the Study } \\
\text { Endpoint }\end{array}$ & $\begin{array}{l}\text { Follow-up } \\
\text { Period } \\
\text { Appropriate } \\
\text { to the Aim } \\
\text { of the Study }\end{array}$ & $\begin{array}{c}\text { Loss to } \\
\text { Follow Up } \\
\text { Less than } \\
5 \%\end{array}$ & $\begin{array}{c}\text { Prospective } \\
\text { Calculation } \\
\text { of the Study } \\
\text { Size }\end{array}$ & $\begin{array}{c}\text { An } \\
\text { Adequate } \\
\text { Control } \\
\text { Group }\end{array}$ & $\begin{array}{l}\text { Contemporary } \\
\text { Group }\end{array}$ & $\begin{array}{l}\text { Baseline } \\
\text { Equivalence } \\
\text { of Groups }\end{array}$ & $\begin{array}{l}\text { Adequate } \\
\text { Statistical } \\
\text { Analyses }\end{array}$ & Tota \\
\hline \multirow{2}{*}{$\begin{array}{c}\text { Slater, 2010 [69] } \\
\text { Maimon, 2013 } \\
\text { [53] }\end{array}$} & Comparative & 2 & 1 & 2 & 2 & 0 & 0 & 0 & 0 & 2 & 2 & 2 & 2 & 15 \\
\hline & Comparative & 2 & 2 & 2 & 1 & 1 & 0 & 0 & 2 & 1 & 1 & 1 & 2 & 15 \\
\hline \multirow{3}{*}{$\begin{array}{l}\text { Fabrizi, , } 2016[71] \\
\text { Hartley, } 2017[55] \\
\text { Verriotis, } 2018 \\
\text { [70] }\end{array}$} & Comparative & 2 & 2 & 2 & 2 & 0 & 0 & 0 & 0 & 1 & 0 & 1 & 2 & 12 \\
\hline & Comparative & 2 & 1 & 2 & 1 & 0 & 0 & 1 & 2 & 1 & 0 & 1 & 2 & 13 \\
\hline & Comparative & 2 & 2 & 2 & 2 & 0 & 0 & 0 & 0 & 1 & 2 & 1 & 2 & 14 \\
\hline Fabrizi, 2011 [72] & $\begin{array}{l}\text { Non- } \\
\text { comparative }\end{array}$ & 2 & 2 & 2 & 1 & 0 & 0 & 0 & 0 & - & - & - & - & 7 \\
\hline Worley, 2012 [73] & $\begin{array}{l}\text { Non- } \\
\text { comparative }\end{array}$ & 1 & 0 & 1 & 1 & 0 & 0 & 0 & 0 & - & - & - & - & 3 \\
\hline Hartley, 2015 [31] & $\begin{array}{c}\text { Non- } \\
\text { comparative }\end{array}$ & 2 & 2 & 2 & 1 & 1 & 0 & 0 & 0 & - & - & - & - & 8 \\
\hline $\begin{array}{c}\text { Verriotis, } 2016 \\
{[74]}\end{array}$ & $\begin{array}{l}\text { Non- } \\
\text { comparative }\end{array}$ & 2 & 2 & 2 & 2 & 0 & 0 & 0 & 0 & - & - & - & - & 8 \\
\hline Maitre, 2017 [54] & $\begin{array}{l}\text { Non- } \\
\text { comparative }\end{array}$ & 2 & 2 & 2 & 1 & 0 & 0 & 0 & 2 & - & - & - & - & 9 \\
\hline Jones, 2017 [75] & $\begin{array}{l}\text { Non- } \\
\text { comparative }\end{array}$ & 1 & 1 & 2 & 1 & 1 & 0 & 0 & 0 & - & - & - & - & 6 \\
\hline Green, 2019 [76] & $\begin{array}{l}\text { Non- } \\
\text { comparative }\end{array}$ & 2 & 2 & 2 & 1 & 1 & 0 & 1 & 0 & - & - & - & - & 9 \\
\hline
\end{tabular}

RCT: randomized controlled trial.

Table 2. Assessment of the methodological quality of the RCT (Jadad score).

\begin{tabular}{|c|c|c|c|c|c|c|c|}
\hline Author, Year & Randomization & Blinding & Withdrawals and Dropouts & Inclusion/Exclusion Criteria & Adverse Effects & Statistical Analysis & Total \\
\hline Norman, 2008 [67] & 1 & 1 & 0 & 1 & 0 & 1 & 4 \\
\hline Slater, 2010 [66] & 2 & 2 & 1 & 1 & 1 & 1 & 8 \\
\hline Gursul, 2018 [65] & 1 & 0 & 0 & 0 & 0 & 1 & 2 \\
\hline Jones, 2020 [64] & 0 & 0 & 0 & 1 & 0 & 1 & 2 \\
\hline
\end{tabular}

RCT: randomized controlled trial. 
Table 3. Summary of included studies (non-RCT).

\begin{tabular}{|c|c|c|c|c|c|c|c|c|}
\hline \multirow[b]{2}{*}{$\begin{array}{l}\text { Author, } \\
\text { Year }\end{array}$} & \multirow[b]{2}{*}{$\begin{array}{c}\text { Study } \\
\text { Design }\end{array}$} & \multicolumn{3}{|c|}{ Participants } & \multirow[b]{2}{*}{ Stimulation } & \multirow[b]{2}{*}{ Outcomes Measured } & \multirow[b]{2}{*}{ Results } & \multirow[b]{2}{*}{${ }^{*}$ Quality } \\
\hline & & Age at Birth & $\begin{array}{l}\text { Age at Time of the } \\
\text { Study }\end{array}$ & Number & & & & \\
\hline $\begin{array}{l}\text { Slater, } 2010 \\
\quad[49]\end{array}$ & $\begin{array}{l}\text { Non- } \\
\text { comparative }\end{array}$ & 35-39 weeks PMA & $2-13$ days & 10 & $\begin{array}{c}\text { Heel lance } \\
\text { Non-noxious control (contact of a } \\
\text { heel lance device against the skin } \\
\text { without the skin being touched by } \\
\text { the blade) }\end{array}$ & ERP & $\begin{array}{l}\text { ERP evoked by heel lance was different from } \\
\text { that by non-noxious control stimulation. }\end{array}$ & $6 / 16$ \\
\hline $\begin{array}{l}\text { Slater, } 2010 \\
\quad[69]\end{array}$ & Comparative & $\begin{array}{c}\text { Term infants: } 37-40 \\
\text { weeks PMA, } \\
\text { Preterm infants: } 24-32 \\
\text { weeks PMA }\end{array}$ & $\begin{array}{l}\text { Term infants: } 37-41 \\
\text { weeks PMA, } \\
\text { Preterm infants: } 37-41 \\
\text { weeks PMA }\end{array}$ & $\begin{array}{c}15 \\
\text { (term infants: } \\
n=8 \\
\text { preterm } \\
\text { infants: } n=7 \text { ) }\end{array}$ & $\begin{array}{c}\text { Heel lance } \\
\text { Lightly tapping a rubber bung }\end{array}$ & ERP & $\begin{array}{l}\text { ERP evoked by heel lance was significantly } \\
\text { larger in preterm infants than term infants. }\end{array}$ & $15 / 24$ \\
\hline $\begin{array}{c}\text { Fabrizi, } \\
2011[72]\end{array}$ & $\begin{array}{l}\text { Non- } \\
\text { comparative }\end{array}$ & 24-42 weeks GA & $28-46$ weeks GA & (2) & $\begin{array}{l}\text { Heel lance } \\
\text { Lightly tapping a tendon hammer }\end{array}$ & ERP & $\begin{array}{l}\text { In full-term infants, ERP evoked by heel lance } \\
\text { (300-700 ms) was different from that by tactile } \\
\text { stimulation ( } 50-300 \mathrm{~ms}) \text {. } \\
\text { The percentage of occurrence of ERP by both } \\
\text { heel lance and tactile stimulation was } \\
\text { significantly smaller in the preterm infants } \\
\text { than the full-term infants. }\end{array}$ & $7 / 16$ \\
\hline $\begin{array}{l}\text { Worley, } \\
2012 \text { [73] }\end{array}$ & $\begin{array}{l}\text { Non- } \\
\text { comparative }\end{array}$ & Infants & No data & 6 & $\begin{array}{c}\text { Heel lance } \\
\text { Lightly tapping a tendon hammer }\end{array}$ & $\begin{array}{c}\text { ERP } \\
\text { EMG } \\
\text { NIRS } \\
\text { ECG } \\
\text { Behavioral responses } \\
\text { Autonomic responses (heart rate, } \\
\text { oxygen saturation, respiratory } \\
\text { rate, and cardiovascular activity) }\end{array}$ & $\begin{array}{c}\text { ERP evoked by heel lance consisted of an early } \\
\text { component followed by a late component. } \\
\text { Tapping stimuli evoked only the early } \\
\text { component. } \\
\text { ERP evoked by heel lance was coupled with } \\
\text { NIRS response. } \\
\text { Only heel lance elicited a larger flexion } \\
\text { withdrawal reflex and behavioral responses, } \\
\text { increased heart rate, and decreased oxygen } \\
\text { saturation. }\end{array}$ & $3 / 16$ \\
\hline $\begin{array}{l}\text { Maimon, } \\
2013[53]\end{array}$ & Comparative & $\begin{array}{l}\text { Group 1: } 27-29 \text { weeks } \\
\text { GA, Group 2: 27-29 } \\
\text { weeks GA, Group 3: } \\
\text { 32-33 weeks GA }\end{array}$ & $\begin{array}{l}\text { Group 1: } 30 \text { weeks GA; } \\
\text { <10 days PNA, Group 2: } \\
33 \text { weeks GA, Group 3: } \\
34.1 \text { weeks GA }\end{array}$ & $\begin{array}{c}\text { Group 1: } \\
n=24 \\
\text { Group 2: } \\
n=22 \\
\text { Group 3: } \\
n=25\end{array}$ & Heel lance & $\begin{array}{l}\text { ERP (evoked power) } \\
\text { Behavioral indicators of infant } \\
\text { pain }\end{array}$ & $\begin{array}{l}\text { No significant difference in brain activity was } \\
\text { found between pre- and post-heel lance. }\end{array}$ & $15 / 24$ \\
\hline $\begin{array}{l}\text { Hartley, } \\
2015[31]\end{array}$ & $\begin{array}{l}\text { Non- } \\
\text { comparative }\end{array}$ & Term infants & $\begin{array}{l}37-42 \text { weeks } G A ;<10 \\
\text { days PNA }\end{array}$ & 30 & $\begin{array}{c}\text { Heel lance } \\
\text { Noxious stimulation (pinprick: } 32 \\
\mathrm{mN}, 64 \mathrm{mN}, 128 \mathrm{mN})\end{array}$ & $\begin{array}{l}\text { ERP } \\
\text { PIPP } \\
\text { EMG }\end{array}$ & $\begin{array}{l}\text { ERP evoked by heel lance ( } 400-700 \mathrm{~ms}) \text { was } \\
\text { greater than that by non-noxious control } \\
\text { stimulation. } \\
\text { The magnitude of ERP was significantly } \\
\text { correlated with the magnitude of EMG. The } \\
\text { ERP and EMG magnitudes increased with } \\
\text { stimulus intensity of pinprick. }\end{array}$ & $8 / 16$ \\
\hline $\begin{array}{l}\text { Verriotis, } \\
2016[74]\end{array}$ & $\begin{array}{l}\text { Non- } \\
\text { comparative }\end{array}$ & $36.3-42.0$ weeks GA & $\begin{array}{l}36.6-43.3 \text { weeks GA; } \\
0-16 \text { days PNA }\end{array}$ & 30 & $\begin{array}{c}\text { Heel lanceControl } \\
\text { stimulation (contact of a heel lance } \\
\text { device against the skin without the } \\
\text { skin being touched by the blade) } \\
\text { Tactile stimulation (lightly tapping } \\
\text { a tendon hammer) }\end{array}$ & $\begin{array}{l}\text { ERP } \\
\text { NIRS }\end{array}$ & $\begin{array}{c}\text { ERP evoked by heel lance consisted of N2P2 } \\
\text { waves ( } 139 \text { and } 202 \mathrm{~ms}) \text { followed by N3P3 } \\
\text { waves ( }(385 \text { and } 554 \mathrm{~ms}) \text {. Tactile stimulation } \\
\text { induced only N2P2 waves ( } 147 \text { and } 248 \mathrm{~ms}) \text {. } \\
\text { Electrophysiological (ERP) and hemodynamic } \\
\text { responses (NIRS) by heel lance coincided and } \\
\text { were positively correlated. }\end{array}$ & $8 / 16$ \\
\hline
\end{tabular}


Table 3. Cont.

\begin{tabular}{|c|c|c|c|c|c|c|c|c|}
\hline \multirow{2}{*}{$\begin{array}{l}\text { Author, } \\
\text { Year }\end{array}$} & \multirow{2}{*}{$\begin{array}{c}\text { Study } \\
\text { Design }\end{array}$} & \multicolumn{3}{|c|}{ Participants } & \multirow[b]{2}{*}{ Stimulation } & \multirow[b]{2}{*}{ Outcomes Measured } & \multirow[b]{2}{*}{ Results } & \multirow{2}{*}{${ }^{*}$ Quality } \\
\hline & & Age at Birth & $\begin{array}{l}\text { Age at Time of the } \\
\text { Study }\end{array}$ & Number & & & & \\
\hline $\begin{array}{l}\text { Fabrizi, } \\
2016[71]\end{array}$ & Comparative & $\begin{array}{c}\text { Infants: } 37-42 \text { weeks } \\
\text { GA, } \\
\text { Adults: - }\end{array}$ & $\begin{array}{c}\text { Infants: } 0-19 \text { days PNA } \\
(5.8 \pm 4.3) \text {, } \\
\text { Adults: } 23-48 \text { years } \\
(29.7 \pm 6.0\end{array}$ & $\begin{array}{l}\text { Infants: } 18, \\
\text { Adults: } 21\end{array}$ & $\begin{array}{c}\text { Infants: Heel lance, } \\
\text { Control stimulation (contact of a } \\
\text { heel lance device against the skin } \\
\text { without the skin being touched by } \\
\text { the blade) } \\
\text { Adults: Noxious stimulation (a } \\
\text { sterile lancet to prick the fifth } \\
\text { finger), } \\
\text { Control stimulation (contact of a } \\
\text { heel lance device against the skin) }\end{array}$ & ERP & $\begin{array}{l}\text { Heel lance (or noxious stimulation) and control } \\
\text { stimulation evoked N2P2 waves in infants ( } 140 \\
\text { and } 225.5 \mathrm{~ms} \text { for heel lance and } 151.5 \text { and } 227 \\
\mathrm{~ms} \text { for control stimulation) and in adults (102 } \\
\text { and } 249.5 \mathrm{~ms} \text { for noxious stimulation and } 93.5 \\
\text { and } 180.5 \mathrm{~ms} \text { for control stimulation). Heel } \\
\text { lance also evoked a N3P3 waves (4003 and } 538 \\
\text { ms) in infants but not in adults. }\end{array}$ & $14 / 24$ \\
\hline $\begin{array}{l}\text { Maitre, } 2017 \\
{[54]}\end{array}$ & $\begin{array}{c}\text { Non- } \\
\text { comparative }\end{array}$ & $37-42$ weeks GA & 2-3 days PNA & 54 & $\begin{array}{l}\text { Heel lance } \\
\text { Light touch (air puff) } \\
\text { Cold puff }\end{array}$ & $\begin{array}{l}\text { ERP } \\
\text { Crying }\end{array}$ & $\begin{array}{l}\text { ERP evoked by heel lance consisted of a late } \\
\text { component. ERP evoked by heel lance was not } \\
\text { associated with either the presence or } \\
\text { amplitude of cries. }\end{array}$ & $9 / 16$ \\
\hline $\begin{array}{c}\text { Jones, } 2017 \\
{[75]}\end{array}$ & $\begin{array}{c}\text { Non- } \\
\text { comparative }\end{array}$ & $36-42$ weeks GA & No data & 56 & Heel lance & $\begin{array}{c}\text { ERP } \\
\text { Salivary cortisol } \\
\text { Heart rate variability } \\
\text { PIPP }\end{array}$ & $\begin{array}{l}\text { ERP evoked by heel lance consisted of N3P3 } \\
\text { waves. ERP amplitude was significantly } \\
\text { correlated with PIPP. }\end{array}$ & $6 / 16$ \\
\hline $\begin{array}{l}\text { Hartley, } \\
2017[55]\end{array}$ & $\begin{array}{l}\text { Non- } \\
\text { comparative }\end{array}$ & 31.9-41.4 weeks GA & 35.1-43.6 weeks GA & 72 & $\begin{array}{c}\text { Heel lance } \\
\text { Experimental noxious stimulation } \\
(128 \mathrm{mN} \text {, pinprick; MRC systems) } \\
\text { Experimental tactile stimulation } \\
\text { (modified tendon hammer) } \\
\text { Visual stimulation } \\
\text { Auditory stimulation }\end{array}$ & $\begin{array}{c}\text { ERP } \\
\text { Heart rate } \\
\text { PIPP (facial expression) }\end{array}$ & $\begin{array}{l}\text { ERP evoked by heel lance }(400-700 \mathrm{~ms}) \text { was } \\
\text { different from that evoked by non-noxious } \\
\text { tactile stimulation. }\end{array}$ & $13 / 24$ \\
\hline $\begin{array}{l}\text { Verriotis, } \\
2018[70]\end{array}$ & $\begin{array}{c}\text { Non- } \\
\text { comparative }\end{array}$ & 29-42 weeks GA & $\begin{array}{l}\text { 29-43 weeks GA; } \\
\text { no older than } 2 \text { weeks } \\
\text { PNA }\end{array}$ & 81 & Heel lance & ERP & $\begin{array}{c}\text { ERP evoked by heel lance consisted of N2P2 } \\
\text { waves followed by N3P3 waves (400-700 ms). } \\
\text { Females were more likely to exhibit a } \\
\text { widespread ERP than males. }\end{array}$ & $14 / 24$ \\
\hline $\begin{array}{c}\text { Jones, } 2018 \\
{[56]}\end{array}$ & $\begin{array}{c}\text { Non- } \\
\text { comparative }\end{array}$ & 23-42 weeks GA & $\begin{array}{c}\text { 29-47 weeks GA (0-96 } \\
\text { days) }\end{array}$ & 112 & $\begin{array}{l}\text { Heel lance } \\
\text { Non-noxious sham (contact of a } \\
\text { heel lance device against the skin } \\
\text { without the skin being touched by } \\
\text { the blade } \\
\text { Auditory controls }\end{array}$ & $\begin{array}{l}\text { ERP } \\
\text { Facial expression } \\
\text { Heart rate } \\
\text { Oxygenation } \\
\text { PIPP }\end{array}$ & $\begin{array}{l}\text { ERP evoked by heel lance consisted of N2P2 } \\
\text { waves followed by N3P3 waves. ERP evoked } \\
\text { by non-noxious sham and auditory control } \\
\text { consisted of N2P2 waves only. }\end{array}$ & $5 / 16$ \\
\hline $\begin{array}{c}\text { Green, } 2019 \\
{[76]}\end{array}$ & $\begin{array}{l}\text { Non- } \\
\text { comparative }\end{array}$ & 23-42 weeks GA & 28-42 weeks GA & 49 & $\begin{array}{c}\text { Heel lance } \\
\text { Control lance (contact of a heel } \\
\text { lance device against the skin } \\
\text { without the skin being touched by } \\
\text { the blade) }\end{array}$ & $\begin{array}{l}\text { PIPP-R (facial expression) } \\
\text { ERP }\end{array}$ & $\begin{array}{l}\text { ERP evoked by heel lance was different from } \\
\text { that by non-noxious control stimulation. The } \\
\text { occurrence of ERP evoked by heel lance } \\
\text { increased with GA. }\end{array}$ & $9 / 16$ \\
\hline
\end{tabular}

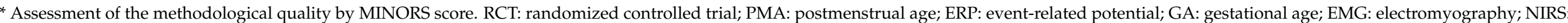
near-infrared spectroscopy; ECG: electrocardiography; N: newton; PNA: postnatal age; PIPP: Premature Infant Pain Profile; PIPP-R: Premature Infant Pain Profile—Revised. 
Table 4. Summary of included studies (RCT).

\begin{tabular}{|c|c|c|c|c|c|c|c|c|}
\hline \multirow[b]{2}{*}{$\begin{array}{l}\text { Author, } \\
\text { Year }\end{array}$} & \multicolumn{3}{|c|}{ Participants } & \multirow[b]{2}{*}{ Stimulation } & \multirow[b]{2}{*}{ Intervention } & \multirow[b]{2}{*}{ Outcomes Measured } & \multirow[b]{2}{*}{ Results } & \multirow[b]{2}{*}{$\begin{array}{l}\text { * Quality } \\
\text { Score }\end{array}$} \\
\hline & Age at Birth & $\begin{array}{l}\text { Age at Time of } \\
\text { the Study }\end{array}$ & Number & & & & & \\
\hline $\begin{array}{l}\text { Norman } \\
2008[67]\end{array}$ & 37-42 weeks GA & 37-143 h PNA & 72 & $\begin{array}{l}\text { Heel lance } \\
\text { Heel prick (non-skin-breaking } \\
\text { pin-prick) } \\
\text { Venous blood sampling from } \\
\text { the dorsum of the hand }\end{array}$ & $\begin{array}{l}\text { (Only heel prick) } \\
\text { Glucose } \\
\text { Water }\end{array}$ & ERP (evoked power)PIPP & $\begin{array}{c}\text { All noxious stimuli induced a significant } \\
\text { increase in higher frequency components } \\
(10-30 \mathrm{~Hz}) \text {. (Only heel prick) } \\
\text { There was no difference in brain activity } \\
\text { between infants who received either glucose or } \\
\text { water. The PIPP score was significantly lower } \\
\text { in infants who received glucose than those } \\
\text { who received water. }\end{array}$ & 4 \\
\hline $\begin{array}{c}\text { Slater } 2010 \\
{[66]}\end{array}$ & 37-43 weeks PMA & $>8$ days PNA & 44 & $\begin{array}{c}\text { Heel lance } \\
\text { Non-noxious control (contact } \\
\text { of a heel lance device against } \\
\text { the skin) }\end{array}$ & $\begin{array}{c}\text { Sucrose } \\
\text { Sterile water }\end{array}$ & $\begin{array}{l}\text { ERP } \\
\text { PIPP } \\
\text { EMG }\end{array}$ & $\begin{array}{l}\text { ERP evoked by heel lance was significantly } \\
\text { greater than that evoked by non-noxious } \\
\text { control. } \\
\text { ERP and EMG response did not differ } \\
\text { significantly between infants who received } \\
\text { either sucrose or sterile water. The PIPP score } \\
\text { was significantly lower in infants who received } \\
\text { sucrose than those who received sterile water. }\end{array}$ & 8 \\
\hline $\begin{array}{c}\text { Gursul } 2018 \\
\text { [65] }\end{array}$ & 37-42 weeks GA & 1-5 days PNA & 30 & $\begin{array}{c}\text { Heel lance } \\
\text { Experimental noxious } \\
\text { stimulus (128 mN, pinprick; } \\
\text { MRC systems) }\end{array}$ & $\begin{array}{c}\text { C-tactile (CT) optimal touch (brush } \\
\text { velocity } 30 \mathrm{~cm} / \mathrm{s} \text { ) } \\
\mathrm{CT} \text { non-optimal touch (brush } \\
\text { velocity } 30 \mathrm{~cm} / \mathrm{s} \text { ) } \\
\text { No-touch control }\end{array}$ & $\begin{array}{l}\text { ERP } \\
\text { EMG }\end{array}$ & $\begin{array}{l}\text { CT optimal touch significantly reduced ERP } \\
\text { evoked by heel lance, as compared to no-touch } \\
\text { control. CT non-optimal touch did not reduce } \\
\text { ERP evoked by heel lance. }\end{array}$ & 2 \\
\hline $\begin{array}{l}\text { Hartley } \\
2018 \text { [68] }\end{array}$ & 34-42 weeks GA & $\begin{array}{c}\text { 34.3-36.3 weeks } \\
\text { GA } \\
\text { 1-20 PNA }\end{array}$ & 31 & $\begin{array}{c}\text { Heel lance } \\
\text { Non-noxious control (contact } \\
\text { of a heel lance device against } \\
\text { the skin) } \\
\text { Retinopathy of prematurity } \\
\text { screening examination }\end{array}$ & $\begin{array}{l}\text { Morphine } \\
\text { Placebo }\end{array}$ & $\begin{array}{c}\text { ERP } \\
\text { PIPP-R }\end{array}$ & $\begin{array}{l}\text { ERP evoked by heel lance was significantly } \\
\text { greater than that by non-noxious control. ERP } \\
\text { and PIPP-R did not differ between infants who } \\
\text { received either morphine or placebo. }\end{array}$ & 7 \\
\hline $\begin{array}{c}\text { Jones } 2020 \\
\text { [64] }\end{array}$ & 23-41 weeks GA & 0-96 PNA & 27 & Heel lance & $\begin{array}{l}\text { While held by a parent in } \\
\text { skin-to-skin } \\
\text { While held by a parent with } \\
\text { clothing } \\
\text { Not held at all }\end{array}$ & $\begin{array}{c}\text { ERP } \\
\text { Facial response } \\
\text { HR }\end{array}$ & $\begin{array}{l}\text { Heel lance evoked noxious ERP components } \\
\text { (497 to } 755 \mathrm{~ms}) \text {. } \\
\text { ERP was significantly lower in infants held } \\
\text { skin-to-skin compared to those held with } \\
\text { clothes. Facial response score and HR were } \\
\text { higher in infants held with clothing than those } \\
\text { not held or those held skin-to-skin. }\end{array}$ & 2 \\
\hline
\end{tabular}

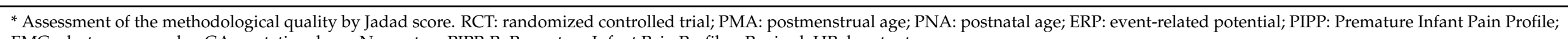
EMG: electromyography; GA: gestational age; N: newton; PIPP-R: Premature Infant Pain Profile—Revised; HR: heart rate. 
An ERP analysis was conducted at electrode $\mathrm{Cz}$ (international 10-20 system) in nine studies [31,55,56,65,66,71,74-76], at electrode Cpz in two studies [72,73], at electrodes Cz and Cpz in four studies $[49,64,69,70]$, and at electrodes C3, C4, F3, and F4 in one study [54]. Two studies did not indicate the electrode location $[67,68]$.

\subsection{ERP Evoked by Heel Lance}

A total of 17 studies demonstrated that heel lance in neonates evoked a specific ERP waveform that consists of both negative and positive peaks [31,49,54-56,64-66,68-76]. One study demonstrated an elevated frequency after heel lance [67], while another did not demonstrate any significant change in frequency [53].

ERP response evoked by heel lance was correlated with PIPP score [75] and EMG response [31] and did coincide with NIRS [73,74]. It did not coincide with crying [54]. When low-intensity pinprick noxious stimulation was applied to infants, ERP response was significantly different from background data, but no difference was noted in PIPP score [31].

\subsection{Comparison of ERP Evoked by Heel Lance and Tactile Stimulation}

Six studies identified distinct negative (N2) and positive (P2) waves after tactile stimulation (between 100 and $400 \mathrm{~ms}$ ) [56,69,71-74]. Ten studies demonstrated that heel lance produced negative (N3) and positive (P3) waves (late component) (between 300 and $700 \mathrm{~ms}$ ) following N2P2 waves (early component) [49,55,56,69-75]. Three of the studies provided details of latency, with 420 and $560 \mathrm{~ms}$ for negative and positive waves, respectively [49], 383 and $554 \mathrm{~ms}$ for negative and positive waves, respectively [74], and 403 and $538 \mathrm{~ms}$ for negative and positive waves, respectively [71]. Seven studies reported ERP components only in a period of 400-755 ms after stimulation with heel lance [31,54,64-66,68,76].

\subsection{Association between GA and ERP Evoked by Heel Lance}

Four studies reported an association between GA and ERP evoked by heel lance $[69,70,72,76]$. Two of the studies demonstrated that ERP response was less likely to be observed in preterm infants compared with full-term infants (33\% (10/30) at GA $28-36$ weeks, and 63\% (19/30) at GA $37-45$ weeks [72]; $12.5 \%(1 / 8)$ at GA $28-32$ weeks, and $82 \%$ (27/33) in GA 33.9-42 weeks [76]). The occurrence of ERP response increased with GA, and the critical period was GA 35-36 weeks [72]. The remaining two studies did not demonstrate a significant association between GA and ERP; one study identified a distinct ERP response in the youngest neonates of the study cohort [70], while the other demonstrated that the range of ERP was greater in preterm infants compared with full-term infants [69].

\subsection{ERP as an Indicator of the Effect of Pain Relief Strategies against Heel Lance in Neonates}

Five RCTs used ERP to determine the effect of pain relief strategies against heel lance in neonates. These strategies included sucrose [66], glucose [67], C-tactile (CT) optimal touch in comparison to CT non-optimal touch [65], holding by a parent either skin-to-skin or with clothing [64], and morphine [68]. CT optimal touch and skin-to-skin holding by a parent reduced the ERP evoked by heel lance [64,65]. Sucrose reduced PIPP but not ERP evoked by heel lance [66], and morphine was not effective in reducing PIPP-R or ERP [68]. Norman et al. examined the response to heel lance (skin breaking), heel prick (non-skin-breaking pinprick), and blood collection and administered either glucose or water to subjects only in the heel prick experiment [67]. They demonstrated that the administration of glucose lowered PIPP but not ERP in response to heel prick.

\section{Discussion}

In this study, to clarify the possibility of ERP evoked by heel lance in neonates as an index of pain assessment, knowledge acquired by and problems of the methods used in studies on ERP evoked by heel lance in neonates were systematically reviewed, including knowledge about $A \delta$ and $C$ fibers responding to noxious stimuli and $A \beta$ fibers responding 
to non-noxious stimuli. Previous reviews were limited to a scoping review, pointing out problems with the sample size and statistical analysis as study methodology [50], and unsystematic mini-reviews [32-34,36]. To our knowledge, no review has systematically organized the study results of ERP evoked by heel lance in neonates. The present review clarified problems of the results and methods of studies on ERP evoked by heel lance in neonates, which may lead to the development of a pain assessment index for heel lance pain in neonates.

\subsection{Results of ERP Evoked by Heel Lance in Term Infants \\ 4.1.1. Characteristics of ERP Evoked by Heel Lance}

In term infants at 37 weeks or more of GA at the time of participation in the study, noxious stimulation with heel lance induces two specific negative and positive ERP waves. In a study in which ERP evoked by heel lance was compared with that evoked by nonnoxious tactile stimulation, both heel lance and non-noxious tactile stimulation induced negative (N2) and positive (P2) waves at around 100-400 ms (early components). In addition to these, heel lance induced negative and positive waves at around 300-700 ms (late components) $[49,55,56,69-75]$. Regarding the late ERP waves evoked by heel lance, Verriotis et al. [74] described that N3P3 is a heel lance-specific ERP following the early components (N2P2), and later studies also reported N3P3 in ERP evoked by heel lance [55, $56,70,75]$. When heel lance was applied to the same neonate at two different times, the early (N2P2) and late (N3P3) ERP components were consistently observed at both times [49]. On the other hand, seven studies reported that heel lance evoked ERP waves only around 400-755 ms [31,54,64-66,68,76]. As the latency of these ERP waves was close to that of N3P3 in other studies, they are likely to be the late components (N3P3). Therefore, noxious heel lance and non-noxious tactile stimulation may be distinguished by the late components (N3P3) in neonates.

\subsubsection{Question about Latency of ERP Evoked by Heel Lance in Neonates}

Although ERP waves have been demonstrated to be evoked by heel lance in a relatively larger number of previous studies, a question remains with regard to the latency of N3P3. In a study in which lance stimulation was applied to adults using the same lance device as that used for heel lance in neonates, the latency of lance stimulation-evoked ERPs was around 100-130 ms for N2 and around $250 \mathrm{~ms}$ for P2 (N2: $102 \mathrm{~ms}, \mathrm{P} 2: 249.5 \mathrm{~ms}$ [71]; N2: $130 \pm 40 \mathrm{~ms}, \mathrm{P} 2: 258 \pm 61 \mathrm{~ms}$ [77]). This latency is extensively short in comparison to that of ERPs evoked by IES, which selectively stimulates the A $\delta$ (N2: 199-232 ms, P2: 302-377 ms) [78-84] and C fibers (P2: 1006-1578 ms) [82,85]. The latency of lance stimulation-evoked ERPs seems to be rather close to that evoked by non-noxious transcutaneous electrical stimulation (ES), which mainly stimulates A $\beta$ fibers (N2: 134-147 ms, P2: 235-293 ms) [78-80]. IES and laser stimulation generate electric currents and selectively stimulate the free nerve ending of A $\delta$ fibers present in the epidermis [80]. The electric current of ES reaches the deeper dermis, where the $A \beta$ receptors are present [80]. Thus, it is possible that heel lance stimulates the $A \beta$ receptors. Specifically, the blade of a lance device used for heel lance reaches a depth of $1 \mathrm{~mm}$ from the skin surface, crossing the $0.2-\mathrm{mm}$ epidermal layer where $\mathrm{A} \delta$ and $C$ fibers reside $[78,86]$, and enters into the dermis (A $\beta$ receptors) [80]. In addition, the lance device is pressed onto the skin surface during lance, which may activate $A \beta$ fibers (tactile pressure or vibration). Accordingly, it cannot be ruled out that lance stimulation activates the $A \beta$ fibers, causing the latency of ERP waves to be closer to that evoked by $\mathrm{A} \beta$-fiber stimulation.

The latency of N3P3 evoked by heel lance in neonates was reported to be $420 \mathrm{~ms}$ [49], $383 \mathrm{~ms}$ [74], and $403 \mathrm{~ms}$ [71] for N3 and $560 \mathrm{~ms}$ [49], $554 \mathrm{~ms}$ [74], and $538 \mathrm{~ms}$ [71] for P3, being longer than that evoked by $\mathrm{A} \delta$ fiber stimulation in adults. However, due to the lower amount of myelination and immature electric current dynamics, neurotransmission of noxious stimuli is slower in neonates than adults $[87,88]$, and there are currently no studies that have specifically examined the latency of ERP evoked by A $\delta$ or $C$ fiber stimulation 
in neonates. Thus, the exact latency evoked by $\mathrm{A} \delta$ or $\mathrm{C}$ fiber stimulation or heel lance in neonates is unclear.

Therefore, we propose the possibility that heel lance-evoked ERPs observed in previous studies reflect the activation of $A \beta$ fibers caused by the blade of the lance device reaching the dermis and/or pressure of the device during lance procedure. Further studies are needed to clarify the latency of ERP evoked by $\mathrm{A} \delta$ and $\mathrm{C}$ fiber stimulations in neonates.

\subsection{Comparison between ERP and PIPP}

PIPP has been used to assess heel lance pain in neonates in combination with facial expression and physiological index in many previous studies. The PIPP provides a heel lance pain score [55,56,66-68], and the PIPP score and the amplitude of ERP (N3P3) evoked by heel lance were positively correlated [75]. However, it has been pointed out that the PIPP may not be sensitive enough to detect low-intensity stimuli compared to ERP [31]. When an experimental pinprick stimulation (the force of 32,64 , and $128 \mathrm{mN}$ ) was used, the PIPP score did not significantly differ from the pre-experimental period. On the other hand, ERP was evoked by all types of experimental pinprick stimulation, and it was significantly different from the pre-experimental period. This result may indicate that ERP has a higher detection sensitivity for low-intensity stimuli than PIPP. However, the latency of ERP evoked by heel lance in neonates questions the activation of $\mathrm{A} \delta$ and/or $\mathrm{C}$ fibers. It remains unclear at this time whether ERP is more sensitive than PIPP.

\subsection{ERP-Based Evaluation of Intervention Effect}

In studies using ERP amplitude as an index of pain assessment, CT optimal touch [65] and holding by a parent, skin-to-skin [64], were found to significantly reduce the N3P3 amplitude of ERP evoked by heel lance in neonates. N3P3 is a nociceptive stimulationspecific ERP because it is evoked by heel lance but not by tactile stimulation. Therefore, the decrease in the N3P3 amplitude could indicate that CT optimal touch and skin-to-skin holding by a parent are useful methods to manage heel lance pain in neonates. On the other hand, sucrose [66] and morphine [68] do not decrease the N3P3 amplitude; thus, they were not regarded as a method to relieve pain in neonates.

However, there is a possibility that ERP evoked by heel lance in neonates reflects the activation of $A \beta$ fiber. Thus, assessing heel lance pain in neonates using ERP may not be appropriate. It is important to perform a study clarifying the ERP evoked by $\mathrm{A} \delta$ and $\mathrm{C}$ fiber stimulations in neonates.

\subsection{ERP Evoked by Heel Lance in Preterm Infants}

A low detection rate of ERP evoked by heel lance in preterm infants can be problematic. In two previous studies, the occurrence of ERP was significantly lower in preterm infants than full-term infants [72,76] and was found to increase with progression of GA [72]. In preterm infants, neuronal bursts can occur in brain circuitry frequently $[72,89]$. It has been suggested that a transition from non-specific neuronal bursts to specific evoked potentials occurs at 35-37 weeks of GA to achieve a discrimination between touch and nociception [72], indicating that ERP evoked by nociceptive stimuli can be detected in neonates at around 35 weeks GA [72].

On the other hand, one study reported no significant association of the occurrence of ERP with GA [70]. Specifically, the occurrence of ERP at electrodes showing the maximum response $(\mathrm{Cz}, \mathrm{C} 4, \mathrm{C} 3, \mathrm{CP} 4$, and $\mathrm{CP} 3)$ was not significantly associated with $\mathrm{GA}$, but a finding of the maximum response at the vertex electrode $(\mathrm{Cz})$ was consistent with two previous reports [72,76]. The response was also lower in preterm than term infants [70]. It is possible that activation of multiple brain regions by heel lance caused the large response at midline electrodes $[70,90,91]$. In preterm infants, a low occurrence of ERP at the vertex electrode has been suggested to be caused by an immaturity of brain, but there are only a few reports that investigated ERPs in preterm infants, necessitating further studies [70]. 
It has also been reported that ERP evoked by heel lance was larger in preterm infants compared to term infants. One study demonstrated that the amplitude of ERPs evoked by heel lance was greater in preterm infants hospitalized in the NICU for at least 40 days than healthy term infants [69]. On the other hand, the response to light touch was found to be smaller in preterm infants than term infants [92]. There are various interpretations of the amplitude ERP evoked by heel lance. Some studies have proposed that the ERP amplitude reflects the intensity of pain perception [93-96]. Preterm infants undergo painful treatments frequently during the NICU stay and thus may be more responsive to noxious heel lance and less responsive to non-invasive light touch [92]. In other words, abnormal neurotransmission develops as a consequence of frequent painful treatments in preterm infants. On the other hand, it is possible that ERP amplitude does not reflect the intensity of pain perception but rather captures the magnitude of attention to a stimulus [97,98]. Nevertheless, ERP studies for preterm infants are at the developmental stage, leaving many unclear points. To advance ERP research in preterm infants, it is necessary to clarify activity of pain-sensing neurons and their association with ERP evoked by heel lance firstly in term infants.

\subsection{Problems of ERP Study Methods in Neonates}

A review of pain assessment using neurophysiological measurements (ERP, NIRS, and functional magnetic resonance imaging (fMRI)) for invasive procedures such as heel lance, intramuscular injections, and noxious pinprick stimulation in neonates highlighted some concerns about the sample sizes of ERP studies [50]. Specifically, the authors demonstrated that only two of eight studies reported sample size calculations, suggesting the need for proper sample size calculations [50]. Similarly, we also demonstrated that only 3 of 13 studies reported sample size calculations. These findings highlight the need for appropriate sample size calculations.

In general, studies on ERP report an average from multiple stimulation trials, with $n=10$ to 12 for intraepidermal electrical stimulation and transcutaneous stimulations $[78,79,82,84]$, to constitute a grand average of the group. On the other hand, the grand average of a single stimulation is calculated for ERP evoked by heel lance in neonates. It is ethically challenging to expose neonates to multiple experimental heel lances, and all of the studies included in the review examined the response to heel lance procedures that were clinically required. Thus, reproducibility of the measurements is a concern in this population [99]. Future studies should address the limitations associated with latency, sample size, and the use of arithmetic means when assessing the level of pain using ERP as an indicator. There is an increasing interest in the brain network associated with pain stimulation in neonates. For example, a study used fMRI to examine the brain activity in response to pinprick in neonates and demonstrated that the areas activated by pain were similar to those in adults [100]. Since ERP can be limited to a few electrodes, it could be beneficial to examine the entire brain network in future studies.

Therefore, problems also remain in the method of studies on ERP evoked by heel lance in neonates, and it is necessary to perform a study with a sufficient sample size and multiple heel lance stimulations.

There are several limitations to our review. First, since only three studies reported sample size calculations, most of the results were not supported by a validated sample size. Exclusion of studies that were not written in English may also have an impact on the overall results of the review.

\section{Conclusions}

ERP evoked by heel lance in neonates consists of N2P2 and N3P3 waves, and N3P3 appears to be induced specifically by heel lance. However, it is possible that N3P3 reflects the activation of $A \beta$ fibers responding to non-noxious stimuli. Furthermore, there are methodological problems such as non-calculated sample size and small number of stimulation trials to obtain an averaged ERP. To establish ERP evoked by heel lance as an index of pain assessment in neonates, it is necessary to clarify ERP evoked by A $\delta$ - and C-fiber stimulations accompanied by heel lance in term infants. A better understanding of this 
aspect may lead to the development of a method reliving heel lance pain and of a pain assessment index for preterm infants.

Author Contributions: Conceptualization, Y.S., M.O. and I.S.; data curation, Y.S., H.U. and K.Y.; writing—original draft preparation, Y.S.; writing—review and editing, H.K., M.O., T.W. and I.S.; supervision, H.K. and I.S. All authors have read and agreed to the published version of the manuscript.

Funding: This research was funded by the Japan Society for the Promotion of Science (JSPS) KAKENHI, grant number JP17K19818 and JP20K19132.

Institutional Review Board Statement: Not applicable.

Informed Consent Statement: Not applicable.

Data Availability Statement: Data sharing not applicable.

Conflicts of Interest: The authors declare no conflict of interest.

\section{References}

1. Cruz, M.D.; Fernandes, A.M.; Oliveira, C.R. Epidemiology of painful procedures performed in neonates: A systematic review of observational studies. Eur. J. Pain 2016, 20, 489-498. [CrossRef] [PubMed]

2. Ranger, M.; Grunau, R.E. Early repetitive pain in preterm infants in relation to the developing brain. Pain Manag. 2014,4 , 57-67. [CrossRef] [PubMed]

3. Brummelte, S.; Grunau, R.E.; Chau, V.; Poskitt, K.J.; Brant, R.; Vinall, J.; Gover, A.; Synnes, A.R.; Miller, S.P. Procedural pain and brain development in premature newborns. Ann. Neurol. 2012, 71, 385-396. [CrossRef]

4. Lax, I.D.; Duerden, E.G.; Lin, S.Y.; Mallar Chakravarty, M.; Donner, E.J.; Lerch, J.P.; Taylor, M.J. Neuroanatomical consequences of very preterm birth in middle childhood. Brain Struct. Funct. 2013, 218, 575-585. [CrossRef]

5. Nosarti, C.; Giouroukou, E.; Healy, E.; Rifkin, L.; Walshe, M.; Reichenberg, A.; Chitnis, X.; Williams, S.C.R.; Murray, R.M. Grey and white matter distribution in very preterm adolescents mediates neurodevelopmental outcome. Brain 2008, 131, 205-217. [CrossRef] [PubMed]

6. Nosarti, C.; Al-Asady, M.H.S.; Frangou, S.; Stewart, A.L.; Rifkin, L.; Murray, R.M. Adolescents who were born very preterm have decreased brain volumes. Brain 2002, 125, 1616-1623. [CrossRef]

7. Grunau, R.E.; Whitfield, M.F.; Petrie-Thomas, J.; Synnes, A.R.; Cepeda, I.L.; Keidar, A.; Rogers, M.; MacKay, M.; Hubber-Richard, P.; Johannesen, D. Neonatal pain, parenting stress and interaction, in relation to cognitive and motor development at 8 and 18 months in preterm infants. Pain 2009, 143, 138-146. [CrossRef]

8. Vinall, J.; Miller, S.P.; Bjornson, B.H.; Fitzpatrick, K.P.V.; Poskitt, K.J.; Brant, R.; Synnes, A.R.; Cepeda, I.L.; Grunau, R.E. Invasive procedures in preterm children: Brain and cognitive development at school age. Pediatrics 2014, 133, 412-421. [CrossRef]

9. Vinall, J.; Miller, S.P.; Synnes, A.R.; Grunau, R.E. Parent behaviors moderate the relationship between neonatal pain and internalizing behaviors at 18 months corrected age in children born very prematurely. Pain 2013, 154, 1831-1839. [CrossRef]

10. Walker, S.M.; Melbourne, A.; O’Reilly, H.; Beckmann, J.; Eaton-Rosen, Z.; Ourselin, S.; Marlow, N. Somatosensory function and pain in extremely preterm young adults from the UK EPICure cohort: Sex-dependent differences and impact of neonatal surgery. Br. J. Anaesth. 2018, 121, 623-635. [CrossRef]

11. Anderson, P.; Doyle, L.W. Neurobehavioral Outcomes of School-age Children Born Extremely Low Birth Weight or Very Preterm in the 1990s. J. Am. Med. Assoc. 2003, 289, 3264-3272. [CrossRef] [PubMed]

12. Loe, I.M.; Lee, E.S.; Luna, B.; Feldman, H.M. Behavior problems of 9-16year old preterm children: Biological, sociodemographic, and intellectual contributions. Early Hum. Dev. 2011, 87, 247-252. [CrossRef] [PubMed]

13. Grunau, R.E.; Whitfield, M.F.; Fay, T.B. Psychosocial and academic characteristics of extremely low birth weight ( $\leq 800$ g) adolescents who are free of major impairment compared with term-born control subjects. Pediatrics 2004, 114, e725-e732. [CrossRef]

14. Schmidt, L.A.; Miskovic, V.; Boyle, M.; Saigal, S. Frontal electroencephalogram asymmetry, salivary cortisol, and internalizing behavior problems in young adults who were born at extremely low birth weight. Child Dev. 2010, 81, 183-199. [CrossRef] [PubMed]

15. Dührsen, L.; Simons, S.H.P.; Dzietko, M.; Genz, K.; Bendix, I.; Boos, V.; Sifringer, M.; Tibboel, D.; Felderhoff-Mueser, U. Effects of repetitive exposure to pain and morphine treatment on the neonatal rat brain. Neonatology 2012, 103, 35-43. [CrossRef]

16. Harrison, D.; Loughnan, P.; Manias, E.; Johnston, L. Analgesics administered during minor painful procedures in a cohort of hospitalized infants: A prospective clinical audit. J. Pain 2009, 10, 715-722. [CrossRef]

17. Bellieni, C.V.; Tei, M.; Cornacchione, S.; Di Lucia, S.; Nardi, V.; Verrotti, A.; Buonocore, G. Pain perception in NICU: A pilot questionnaire. J. Matern. Fetal Neonatal Med. 2018, 31, 1921-1923. [CrossRef]

18. Pillai Riddell, R.; Racine, N.; Gennis, H.; Turcotte, K.; Uman, L.; Horton, R.; Ahola Kohut, S.; Hillgrove Stuart, J.; Stevens, B.; Lisi, D. Non-pharmacological management of infant and young child procedural pain (Review) summary of findings for the main comparison. Cochrane Database Syst. Rev. 2015. [CrossRef] 
19. Stevens, B.; Yamada, J.; Ohlsson, A.; Haliburton, S.; Shorkey, A. Sucrose for analgesia in newborn infants undergoing painful procedures (Review) SUMMARY OF FINDINGS FOR THE MAIN COMPARISON. Cochrane Libr. 2017. [CrossRef]

20. Campbell-Yeo, M. Combining facilitated tucking and non-nutritive sucking appears to promote greater regulation for preterm neonates following heel lance, but does not provide effective pain relief. Evid. Based Nurs. 2019, 22, 19. [CrossRef]

21. Gao, H.; Li, M.; Gao, H.; Xu, G.; Li, F.; Zhou, J.; Zou, Y.; Jiang, H. Effect of non-nutritive sucking and sucrose alone and in combination for repeated procedural pain in preterm infants: A randomized controlled trial. Int. J. Nurs. Stud. 2018, 83, 25-33. [CrossRef] [PubMed]

22. Peng, H.F.; Yin, T.; Yang, L.; Wang, C.; Chang, Y.C.; Jeng, M.J.; Liaw, J.J. Non-nutritive sucking, oral breast milk, and facilitated tucking relieve preterm infant pain during heel-stick procedures: A prospective, randomized controlled trial. Int. J. Nurs. Stud. 2018, 77, 162-170. [CrossRef] [PubMed]

23. Perroteau, A.; Nanquette, M.C.; Rousseau, A.; Renolleau, S.; Bérard, L.; Mitanchez, D.; Leblanc, J. Efficacy of facilitated tucking combined with non-nutritive sucking on very preterm infants' pain during the heel-stick procedure: A randomized controlled trial. Int. J. Nurs. Stud. 2018, 86, 29-35. [CrossRef] [PubMed]

24. Davari, S.; Borimnejad, L.; Khosravi, S.; Haghani, H. The effect of the facilitated tucking position on pain intensity during heel stick blood sampling in premature infants: A surprising result. J. Matern. Neonatal Med. 2019, 32, 3427-3430. [CrossRef]

25. Uematsu, H.; Sobue, I. Effect of music (brahms lullaby) and non-nutritive sucking on heel lance in preterm infants: A randomized controlled crossover trial. Paediatr. Child Health 2019, 24, E33-E39. [CrossRef]

26. Ballantyne, M.; Stevens, B.; McAllister, M.; Dionne, K.; Jack, A. Validation of the premature infant pain profile in the clinical setting. Clin. J. Pain 1999, 15, 297-303. [CrossRef]

27. Stevens, B.; Johnston, C.; Petryshen, P.; Taddio, A. Premature Infant Pain Profile: Development and initial validation. Clin. J. Pain 1996, 12, 13-22. [CrossRef]

28. Gibbins, S.; Stevens, B.J.; Yamada, J.; Dionne, K.; Campbell-Yeo, M.; Lee, G.; Caddell, K.; Johnston, C.; Taddio, A. Validation of the Premature Infant Pain Profile-Revised (PIPP-R). Early Hum. Dev. 2014, 90, 189-193. [CrossRef]

29. Stevens, B.J.; Gibbins, S.; Yamada, J.; Dionne, K.; Lee, G.; Johnston, C.; Taddio, A. The premature infant pain profile-revised (PIPP-R): Initial validation and feasibility. Clin. J. Pain 2014, 30, 238-243. [CrossRef]

30. Stevens, B.; Johnston, C.; Taddio, A.; Gibbins, S.; Yamada, J. The premature infant pain profile: Evaluation 13 years after development. Clin. J. Pain 2010, 26, 813-830. [CrossRef]

31. Hartley, C.; Goksan, S.; Poorun, R.; Brotherhood, K.; Mellado, G.S.; Moultrie, F.; Rogers, R.; Adams, E.; Slater, R. The relationship between nociceptive brain activity, spinal reflex withdrawal and behaviour in newborn infants. Sci. Rep. 2015, 5, 1-13. [CrossRef] [PubMed]

32. Moultrie, F.; Slater, R.; Hartley, C. Improving the treatment of infant pain. Curr. Opin. Support. Palliat. Care 2017, 11, 112-117. [CrossRef] [PubMed]

33. Shiroshita, Y.; Kirimoto, H.; Nakagawa, K.; Uematsu, H.; Sobue, I. Evoked Potential as a Pain Evaluation Index for Neonatal Procedural Pain. Int. J. Nurs. Clin. Pract. 2020, 7. [CrossRef]

34. Gursul, D.; Hartley, C.; Slater, R. Nociception and the neonatal brain. Semin. Fetal Neonatal Med. 2019, 24, 101016. [CrossRef] [PubMed]

35. Relland, L.M.; Gehred, A.; Maitre, N.L. Behavioral and Physiological Signs for Pain Assessment in Preterm and Term Neonates during a Nociception-Specific Response: A Systematic Review. Pediatr. Neurol. 2019, 90, 13-23. [CrossRef] [PubMed]

36. Hartley, C.; Slater, R. Neurophysiological measures of nociceptive brain activity in the newborn infant-the next steps. Acta Paediatr. Int. J. Paediatr. 2014, 103, 238-242. [CrossRef] [PubMed]

37. Tzabazis, A.Z.; Klukinov, M.; Crottaz-Herbette, S.; Nemenov, M.I.; Angst, M.S.; Yeomans, D.C. Selective nociceptor activation in volunteers by infrared diode laser. Mol. Pain 2011, 7, 1744-8069. [CrossRef] [PubMed]

38. Zotterman, Y. Touch, pain and tickling: An electro-physiological investigation on cutaneous sensory nerves. J. Physiol. 1939, 95, 1-28. [CrossRef]

39. Burgess, P.R.; Perl, E.R. Myelinated afferent fibres responding specifically to noxious stimulation of the skin. J. Physiol. 1967, 190, 541-562. [CrossRef]

40. Torebjörk, H.E.; Hallin, R.G. Perceptual changes accompanying controlled preferential blocking of A and C fibre responses in intact human skin nerves. Exp. Brain Res. 1973, 16, 321-332. [CrossRef]

41. Hallin, R.G.; Torebjörk, H.E. Electrically induced A and C fibre responses in intact human skin nerves. Exp. Brain Res. 1973, 16, 309-320. [CrossRef] [PubMed]

42. Price, D.D.; Barrell, J.J.; Gracely, R.H. A psychophysical analysis of experiential factors that selectively influence the affective dimension of pain. Pain 1980, 8, 137-149. [CrossRef]

43. Adriaensen, H.; Gybels, J.; Handwerker, H.O.; Van Hees, J. Response properties of thin myelinated (A-delta) fibers in human skin nerves. J. Neurophysiol. 1983, 49, 111-122. [CrossRef] [PubMed]

44. Hatfield, L. Neonatal pain: What's age got to do with it? Surg. Neurol. Int. 2014, 5, 479. [CrossRef]

45. Basbaum, A.I.; Fields, H.L. Endogenous pain control systems: Brainstem spinal pathways and endorphin circuitry. Annu. Rev. Neurosci. 1984, 7, 309-338. [CrossRef]

46. Inui, K.; Kakigi, R. Pain perception in humans: Use of intraepidermal electrical stimulation. J. Neurol. Neurosurg. Psychiatry 2012, 83, 551-556. [CrossRef] 
47. Truini, A.; Panuccio, G.; Galeotti, F.; Maluccio, M.R.; Sartucci, F.; Avoli, M.; Cruccu, G. Laser-evoked potentials as a tool for assessing the efficacy of antinociceptive drugs. Eur. J. Pain 2010, 14, 222-225. [CrossRef]

48. Malver, L.P.; Brokjær, A.; Staahl, C.; Graversen, C.; Andresen, T.; Drewes, A.M. Electroencephalography and analgesics. Br. J. Clin. Pharmacol. 2014, 77, 72-95. [CrossRef]

49. Slater, R.; Worley, A.; Fabrizi, L.; Roberts, S.; Meek, J.; Boyd, S.; Fitzgerald, M. Evoked potentials generated by noxious stimulation in the human infant brain. Eur. J. Pain 2010, 14, 321-326. [CrossRef]

50. Benoit, B.; Martin-Misener, R.; Newman, A.; Latimer, M.; Campbell-Yeo, M. Neurophysiological assessment of acute pain in infants: A scoping review of research methods. Acta Paediatr. Int. J. Paediatr. 2017, 106, 1053-1066. [CrossRef]

51. Liberati, A.; Altman, D.G.; Tetzlaff, J.; Mulrow, C.; Gøtzsche, P.C.; Ioannidis, J.P.A.; Clarke, M.; Devereaux, P.J.; Kleijnen, J.; Moher, D. The PRISMA statement for reporting systematic reviews and meta-analyses of studies that evaluate health care interventions: Explanation and elaboration. PLoS Med. 2009, 6, e1000100. [CrossRef] [PubMed]

52. Moher, D.; Liberati, A.; Tetzlaff, J.; Altman, D.G.; Altman, D.; Antes, G.; Atkins, D.; Barbour, V.; Barrowman, N.; Berlin, J.A.; et al. Preferred reporting items for systematic reviews and meta-analyses: The PRISMA statement. PLoS Med. 2009,6 , e1000097. [CrossRef] [PubMed]

53. Maimon, N.; Grunau, R.E.; Cepeda, I.L.; Friger, M.; Selnovik, L.; Gilat, S.; Shany, E. Electroencephalographic activity in response to procedural pain in preterm infants born at 28 and 33 weeks gestational age. Clin. J. Pain 2013, 29, 1044-1049. [CrossRef] [PubMed]

54. Maitre, N.L.; Stark, A.R.; McCoy Menser, C.C.; Chorna, O.D.; France, D.J.; Key, A.F.; Wilkens, K.; Moore-Clingenpeel, M.; Wilkes, D.M.; Bruehl, S. Cry presence and amplitude do not reflect cortical processing of painful stimuli in newborns with distinct responses to touch or cold. Arch. Dis. Child. Fetal Neonatal Ed. 2017, 102, F428-F433. [CrossRef] [PubMed]

55. Hartley, C.; Duff, E.P.; Green, G.; Mellado, G.S.; Worley, A.; Rogers, R.; Slater, R. Nociceptive brain activity as a measure of analgesic efficacy in infants. Sci. Transl. Med. 2017, 9, eaah6122. [CrossRef] [PubMed]

56. Jones, L.; Laudiano-Dray, M.P.; Whitehead, K.; Verriotis, M.; Meek, J.; Fitzgerald, M.; Fabrizi, L. EEG, behavioural and physiological recordings following a painful procedure in human neonates. Sci. Data 2018, 5, 1-10. [CrossRef]

57. Slim, K.; Nini, E.; Forestier, D.; Kwiatkowski, F.; Panis, Y.; Chipponi, J. Methodological index for non-randomized studies (Minors): Development and validation of a new instrument. ANZ J. Surg. 2003, 73, 712-716. [CrossRef]

58. Ma, L.L.; Wang, Y.Y.; Yang, Z.H.; Huang, D.; Weng, H.; Zeng, X.T. Methodological quality (risk of bias) assessment tools for primary and secondary medical studies: What are they and which is better? Mil. Med. Res. 2020, 7, 1-11. [CrossRef]

59. Greenhalgh, T. Assessing the methodological quality of published papers. BMJ 1997, 315, 305-308. [CrossRef]

60. Oremus, M.; Wolfson, C.; Perrault, A.; Demers, L.; Momoli, F.; Moride, Y. Interrater reliability of the modified Jadad quality scale for systematic reviews of Alzheimer's disease drug trials. Dement. Geriatr. Cogn. Disord. 2001, 12, 232-236. [CrossRef]

61. Jadad, A.R.; Moore, R.A.; Carroll, D.; Jenkinson, C.; Reynolds, D.J.M.; Gavaghan, D.J.; McQuay, H.J. Assessing the quality of reports of randomized clinical trials: Is blinding necessary? Control. Clin. Trials 1996, 17, 1-12. [CrossRef]

62. Wu, P.L.; Lee, M.; Huang, T.T. Effectiveness of physical activity on patients with depression and Parkinson's disease: A systematic review. PLoS ONE 2017, 12, e0181515. [CrossRef] [PubMed]

63. Crellin, D.J.; Harrison, D.; Santamaria, N.; Babl, F.E. Systematic review of the Face, Legs, Activity, Cry and Consolability scale for assessing pain in infants and children. Pain 2015, 156, 2132-2151. [CrossRef] [PubMed]

64. Jones, L.; Laudiano-Dray, M.P.; Whitehead, K.; Meek, J.; Fitzgerald, M.; Fabrizi, L.; Pillai Riddell, R. The impact of parental contact upon cortical noxious-related activity in human neonates. Eur. J. Pain 2020, 1-11. [CrossRef] [PubMed]

65. Gursul, D.; Goksan, S.; Hartley, C.; Mellado, G.S.; Moultrie, F.; Hoskin, A.; Adams, E.; Hathway, G.; Walker, S.; McGlone, F.; et al. Stroking modulates noxious-evoked brain activity in human infants. Curr. Biol. 2018, 28, R1380-R1381. [CrossRef]

66. Slater, R.; Cornelissen, L.; Fabrizi, L.; Patten, D.; Yoxen, J.; Worley, A.; Boyd, S.; Meek, J.; Fitzgerald, M. Oral sucrose as an analgesic drug for procedural pain in newborn infants: A randomised controlled trial. Lancet 2010, 376, 1225-1232. [CrossRef]

67. Norman, E.; Rosén, I.; Vanhatalo, S.; Stjernqvist, K.; Ökland, O.; Fellman, V.; Hellström-Westas, L. Electroencephalographic response to procedural pain in healthy term newborn infants. Pediatr. Res. 2008, 64, 429-434. [CrossRef]

68. Hartley, C.; Moultrie, F.; Hoskin, A.; Green, G.; Monk, V.; Bell, J.L.; King, A.R.; Buckle, M.; van der Vaart, M.; Gursul, D.; et al. Analgesic efficacy and safety of morphine in the Procedural Pain in Premature Infants (Poppi) study: Randomised placebo-controlled trial. Lancet 2018, 392, 2595-2605. [CrossRef]

69. Slater, R.; Fabrizi, L.; Worley, A.; Meek, J.; Boyd, S.; Fitzgerald, M. Premature infants display increased noxious-evoked neuronal activity in the brain compared to healthy age-matched term-born infants. Neuroimage 2010, 52, 583-589. [CrossRef]

70. Verriotis, M.; Jones, L.; Whitehead, K.; Laudiano-Dray, M.; Panayotidis, I.; Patel, H.; Meek, J.; Fabrizi, L.; Fitzgerald, M. The distribution of pain activity across the human neonatal brain is sex dependent. Neuroimage 2018, 178, 69-77. [CrossRef]

71. Fabrizi, L.; Verriotis, M.; Williams, G.; Lee, A.; Meek, J.; Olhede, S.; Fitzgerald, M. Encoding of mechanical nociception differs in the adult and infant brain. Sci. Rep. 2016, 6, 2-10. [CrossRef] [PubMed]

72. Fabrizi, L.; Slater, R.; Worley, A.; Meek, J.; Boyd, S.; Olhede, S.; Fitzgerald, M. A shift in sensory processing that enables the developing human brain to discriminate touch from pain. Curr. Biol. 2011, 21, 1552-1558. [CrossRef] [PubMed]

73. Worley, A.; Fabrizi, L.; Boyd, S.; Slater, R. Multi-modal pain measurements in infants. J. Neurosci. Methods 2012, $205,252-257$. [CrossRef] [PubMed] 
74. Verriotis, M.; Fabrizi, L.; Lee, A.; Cooper, R.J.; Fitzgerald, M.; Meek, J. Mapping cortical responses to somatosensory stimuli in human infants with simultaneous near-infrared spectroscopy and event-related potential recording. ENeuro 2016, 3, 663-673. [CrossRef] [PubMed]

75. Jones, L.; Fabrizi, L.; Laudiano-Dray, M.; Whitehead, K.; Meek, J.; Verriotis, M.; Fitzgerald, M. Nociceptive Cortical Activity Is Dissociated from Nociceptive Behavior in Newborn Human Infants under Stress. Curr. Biol. 2017, 27, 3846-3851.e3. [CrossRef]

76. Green, G.; Hartley, C.; Hoskin, A.; Duff, E.; Shriver, A.; Wilkinson, D.; Adams, E.; Rogers, R.; Moultrie, F.; Slater, R. Behavioural discrimination of noxious stimuli in infants is dependent on brain maturation. Pain 2019, 160, 493-500. [CrossRef]

77. Fabrizi, L.; Williams, G.; Lee, A.; Meek, J.; Slater, R.; Olhede, S.; Fitzgerald, M. Cortical activity evoked by an acute painful tissue-damaging stimulus in healthy adult volunteers. J. Neurophysiol. 2013, 109, 2393-2403. [CrossRef]

78. Inui, K.; Tran, T.D.; Hoshiyama, M.; Kakigi, R. Preferential stimulation of A $\delta$ fibers by intra-epidermal needle electrode in humans. Pain 2002, 96, 247-252. [CrossRef]

79. Otsuru, N.; Inui, K.; Yamashiro, K.; Miyazaki, T.; Takeshima, Y.; Kakigi, R. Assessing A fiber function with lidocaine using intraepidermal electrical stimulation. J. Pain 2010, 11, 621-627. [CrossRef]

80. Mouraux, A.; Iannetti, G.D.; Plaghki, L. Low intensity intra-epidermal electrical stimulation can activate A $\delta$-nociceptors selectively. Pain 2010, 150, 199-207. [CrossRef]

81. Mouraux, A.; Marot, E.; Legrain, V. Short trains of intra-epidermal electrical stimulation to elicit reliable behavioral and electrophysiological responses to the selective activation of nociceptors in humans. Neurosci. Lett. 2014, 561, 69-73. [CrossRef] [PubMed]

82. Kodaira, M.; Inui, K.; Kakigi, R. Evaluation of nociceptive A $\delta$ - and C-fiber dysfunction with lidocaine using intraepidermal electrical stimulation. Clin. Neurophysiol. 2014, 125, 1870-1877. [CrossRef] [PubMed]

83. Omori, S.; Isose, S.; Misawa, S.; Watanabe, K.; Sekiguchi, Y.; Shibuya, K.; Beppu, M.; Amino, H.; Kuwabara, S. Pain-related evoked potentials after intraepidermal electrical stimulation to A $\delta$ and $C$ fibers in patients with neuropathic pain. Neurosci. Res. 2017, 121, 43-48. [CrossRef] [PubMed]

84. Kirimoto, H.; Tamaki, H.; Otsuru, N.; Yamashiro, K.; Onishi, H.; Nojima, I.; Oliviero, A. Transcranial static magnetic field stimulation over the primary motor cortex induces plastic changes in cortical nociceptive processing. Front. Hum. Neurosci. 2018, 12, 63. [CrossRef] [PubMed]

85. Otsuru, N.; Inui, K.; Yamashiro, K.; Miyazaki, T.; Ohsawa, I.; Takeshima, Y.; Kakigi, R. Selective Stimulation of C Fibers by an Intra-Epidermal Needle Electrode in Humans. Open Pain J. 2009, 2, 53-56. [CrossRef]

86. Novotny, G.E.; Gommert-Novotny, E. Intraepidermal nerves in human digital skin. Cell Tissue Res. 1988, 254, 111-117. [CrossRef]

87. Levitt, P. Structural and functional maturation of the developing primate brain. J. Pediatr. 2003, 143, 35-45. [CrossRef]

88. Takahashi, T. Postsynaptic receptor mechanisms underlying developmental speeding of synaptic transmission. Neurosci. Res. 2005, 53, 229-240. [CrossRef]

89. Khazipov, R.; Luhmann, H.J. Early patterns of electrical activity in the developing cerebral cortex of humans and rodents. Trends Neurosci. 2006, 29, 414-418. [CrossRef]

90. Kucyi, A.; Davis, K.D. The dynamic pain connectome. Trends Neurosci. 2015, 38, 86-95. [CrossRef]

91. Apkarian, A.V.; Bushnell, M.C.; Treede, R.D.; Zubieta, J.K. Human brain mechanisms of pain perception and regulation in health and disease. Eur. J. Pain 2005, 9, 463. [CrossRef]

92. Maitre, N.L.; Key, A.P.; Chorna, O.D.; Slaughter, J.C.; Matusz, P.J.; Wallace, M.T.; Murray, M.M. The Dual Nature of Early-Life Experience on Somatosensory Processing in the Human Infant Brain. Curr. Biol. 2017, 27, 1048-1054. [CrossRef]

93. Iannetti, G.D.; Zambreanu, L.; Cruccu, G.; Tracey, I. Operculoinsular cortex encodes pain intensity at the earliest stages of cortical processing as indicated by amplitude of laser-evoked potentials in humans. Neuroscience 2005, 131, 199-208. [CrossRef] [PubMed]

94. Ohara, S.; Crone, N.E.; Weiss, N.; Treede, R.D.; Lenz, F.A. Amplitudes of laser evoked potential recorded from primary somatosensory, parasylvian and medial frontal cortex are graded with stimulus intensity. Pain 2004, 110, 318-328. [CrossRef] [PubMed]

95. García-larrea, L.; Peyron, R.; Laurent, B.; Mauguière, F.; Bernard, U.C.; Neurologie, D.; Bellevue, H.; Etienne, S. garcia_Larrea97. Neuroreport 1997, 8, 1-5.

96. Beydoun, A.; Morrow, T.J.; Shen, J.F.; Casey, K.L. Variability of laser-evoked potentials: Attention, arousal and lateralized differences. Electroencephalogr. Clin. Neurophysiol. Evoked Potentials 1993, 88, 173-181. [CrossRef]

97. Legrain, V.; Mancini, F.; Sambo, C.F.; Torta, D.M.; Ronga, I.; Valentini, E. Cognitive aspects of nociception and pain. Bridging neurophysiology with cognitive psychology. Neurophysiol. Clin. 2012, 42, 325-336. [CrossRef] [PubMed]

98. Iannetti, G.D.; Hughes, N.P.; Lee, M.C.; Mouraux, A. Determinants of laser-evoked EEG responses: Pain perception or stimulus saliency? J. Neurophysiol. 2008, 100, 815-828. [CrossRef]

99. Baumgärtner, U.; Greffrath, W.; Treede, R.D. Contact heat and cold, mechanical, electrical and chemical stimuli to elicit small fiber-evoked potentials: Merits and limitations for basic science and clinical use. Neurophysiol. Clin. 2012, 42, 267-280. [CrossRef]

100. Goksan, S.; Hartley, C.; Emery, F.; Cockrill, N.; Poorun, R.; Moultrie, F.; Rogers, R.; Campbell, J.; Sanders, M.; Adams, E.; et al. fMRI reveals neural activity overlap between adult and infant pain. Elife 2015, 4, e06356. [CrossRef] 